Pharmaceutical Nanotechnology

\title{
Design of cationic lipid nanoparticles for ocular delivery: Development, characterization and cytotoxicity
}

\author{
Joana F. Fangueiro a ${ }^{\text {, Tatiana Andreani }}{ }^{\mathrm{a}, \mathrm{b}, \mathrm{c}}$, Maria A. Egea ${ }^{\mathrm{d}, \mathrm{e}}$, Maria L. Garcia $^{\mathrm{d}, \mathrm{e}}$, \\ Selma B. Souto ${ }^{\mathrm{f}}$, Amélia M. Silva ${ }^{\mathrm{b}, \mathrm{c}}$, Eliana B. Souto ${ }^{\mathrm{a}, \mathrm{g}, *}$ \\ ${ }^{a}$ Faculty of Health Sciences, Fernando Pessoa University (UFP-FCS), Rua Carlos da Maia, 296, 4200-150 Porto, Portugal \\ ${ }^{\mathrm{b}}$ Centre for Research and Technology of Agro-Environmental and Biological Sciences (CITAB), Vila Real, Portugal

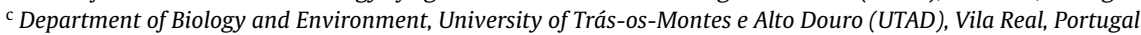 \\ d Department of Physical Chemistry, Faculty of Pharmacy, University of Barcelona, Av. Joan XXIII s/n, 08028 Barcelona, Spain \\ ${ }^{\mathrm{e}}$ Institute of Nanoscience and Nanotechnology, University of Barcelona, Av. Joan XXIII s/n, 08028 Barcelona, Spain \\ ${ }^{\mathrm{f}}$ Division of Endocrinology, Diabetes and Metabolism, Hospital de Braga, Braga, Portugal \\ $\mathrm{g}$ Institute of Biotechnology and Bioengineering, Centre of Genetics and Biotechnology, Trás-os-Montes and Alto Douro University (IBB/CGB-UTAD), \\ Vila Real, Portugal
}

\section{A R T I C L E I N F O}

\section{Article history:}

Received 11 October 2013

Received in revised form

10 November 2013

Accepted 15 November 2013

Available online 23 November 2013

\section{Keywords:}

Lipid nanoparticles

Ocular delivery

Multiple emulsion

Cytotoxicity

Turbiscan

Y-79 human retinoblastoma cells

\begin{abstract}
A B S T R A C T
In the present study we have developed lipid nanoparticle (LN) dispersions based on a multiple emulsion technique for encapsulation of hydrophilic drugs or/and proteins by a full factorial design. In order to increase ocular retention time and mucoadhesion by electrostatic attraction, a cationic lipid, namely cetyltrimethylammonium bromide (CTAB), was added in the lipid matrix of the optimal LN dispersion obtained from the factorial design. There are a limited number of studies reporting the ideal concentration of cationic agents in LN for drug delivery. This paper suggests that the choice of the concentration of a cationic agent is critical when formulating a safe and stable LN. CTAB was included in the lipid matrix of $\mathrm{LN}$, testing four different concentrations $(0.25 \%, 0.5 \%, 0.75 \%$, or $1.0 \% \mathrm{wt})$ and how composition affects LN behavior regarding physical and chemical parameters, lipid crystallization and polymorphism, and stability of dispersion during storage. In order to develop a safe and compatible system for ocular delivery, CTAB-LN dispersions were exposed to Human retinoblastoma cell line Y-79. The toxicity testing of the CTAB-LN dispersions was a fundamental tool to find the best CTAB concentration for development of these cationic $\mathrm{LN}$, which was found to be $0.5 \mathrm{wt} \%$ of CTAB.
\end{abstract}

(c) 2013 Elsevier B.V. All rights reserved.

\section{Introduction}

Lipid nanoparticles (LN) have gained interest in recent years as drug carriers for ocular delivery, aiming a better permeation and/or prolonged drug release onto the ocular mucosa and allowing drugs reaching the post segment of the eye (Pignatello and Puglisi, 2011). Ocular drug delivery is extremely affected by eye anatomy and physiology that leads often to mechanisms that decrease bioavailability of applied drugs. These mechanisms include reflex processes, such as lacrimation and blinking which reduces drastically the drug residence time, and difficulty to diffuse though the conjunctiva and nasolacrimal duct. In addition, the low volume of the conjunctival sac also leads to a poor corneal or sclera penetration of drugs (Diebold and Calonge, 2010). Since ocular delivery

\footnotetext{
* Corresponding author at: Faculty of Health Sciences of Fernando Pessoa University, Rua Carlos da Maia, 296, Office S.1, P-4200-150 Porto, Portugal. Tel.: +351 22507 4630x3056; fax: +351225504637.

E-mail addresses: eliana@ufp.edu.pt, souto.eliana@gmail.com (E.B. Souto).
}

became a problem when the ultimate target is intraocular delivery, due to the ineffective drug concentrations and time residence reach the inner tissues, alternative systems for drug delivery are required (Pignatello and Puglisi, 2011; Sultana et al., 2011). New drug delivery systems based on lipids, namely liposomes, and other materials such as polymers (poly-D-L-lactic acid (PLA) nanopsheres) were able to deliver an antiviral drug, acyclovir, in the inner tissues of the eye comprising the innovation of these systems (Fresta et al., 1999; Giannavola et al., 2003).

Ocular drug delivery strategies may be classified into 3 groups: noninvasive techniques, implants, and colloidal carriers. Colloidal drug delivery systems, such as LN, can be easily administered in a liquid form and have the ability to diffuse rapidly and are better internalized in ocular tissues. In addition, the interaction and adhesion of LN ocular surface with the endothelium makes these drug delivery systems interesting as new therapeutic tools in ocular delivery (del Pozo-Rodriguez et al., 2013).

LN based on $\mathrm{w} / \mathrm{o} / \mathrm{w}$ emulsion are versatile colloidal carriers for the administration of peptides/proteins and hydrophilic drugs (Fangueiro et al., 2012). Droplets from the inner aqueous phase, 
where the drug is dissolved or/and solubilized, are supported by a solid lipid matrix surrounded by an aqueous surfactant phase. Usually LN are composed of physiological solid lipids (mixtures of mono-, di- or triglycerides, fatty acids or waxes) stabilized by surfactants. In the case of a w/o/w based LN dispersion, a high hydrophilic-lipophilic balance (HLB) surfactant is added to the external aqueous phase and, a low HLB surfactant is added to the lipid phase. The two surfactants are needed to stabilize the two existing interfaces in this type of emulsion. A variety of surfactants can be applied, such as phospholipids, bile salts, polysorbates, polyoxyethylene ethers (Gallarate et al., 2009; Fangueiro et al., 2012). Materials used for LN production are largely used in pharmaceutical industry with proved biocompatibility (Severino et al., 2012).

Cationic LN have been recently investigated for targeting ocular mucosa, namely the posterior segment of the eye (e.g. retina). This is a smart strategy that combines the positive surface charge of the particles and the negative surface charge of ocular mucosa by means of an electrostatic attraction. This approach could increase the drugs retention time in the eye as well as improve nanoparticles bioadhesion (Lallemand et al., 2012).

In the ocular delivery, it is especially relevant the control of the particle size since it directly influence the drug release rate, bioavailability, and patient comfort and compliance (Shekunov et al., 2007; Souto et al., 2010). In addition, it is known that the smaller the particle size, the longer the retention time and easier application (Araujo et al., 2009).

Physicochemical characterization and assessment of nanotoxicity are major issues for developing and large-scale manufacturing of nanocarriers. Furthermore, physicochemical properties of LN such as particle size, surface and composition can significantly influence drug delivery on ocular delivery (Ying et al., 2013).

In the present work, the development and characterization of a system of LN based on multiple emulsions using a blend of triacylglycerols as solid lipid, was carried out, in which sonication method was employed. The first aim of the work was the application of a full factorial design to determine which dependent variables could affect the LN dispersion properties. The analyzed independent variables, namely the concentration of solid lipid and both hydrophilic and lipophilic surfactants, were checked for their capacity to influence the mean particle size (Z-Ave), polydispersity index (PI) and zeta potential (ZP) of the produced LNs dispersions. The optimal formulation was used to evaluate the toxicity of LN using Y-79 human retinoblastoma cells employing different cationic lipid concentrations to select the best formulation for ocular instillations.

\section{Materials and methods}

\subsection{Materials}

Softisan ${ }^{\circledR} 100$ (S100, a hydrogenated coco-glycerides $C_{10}-C_{18}$ fatty acid triacylglycerol) used as solid lipid was a free sample from Sasol Germany GmbH (Witten, Germany), Lipoid ${ }^{\circledR}$ S75, 75\% soybean phosphatidylcholine, used as surfactant, was purchased from Lipoid GmbH (Ludwigshafen, Germany), Lutrol ${ }^{\circledR}$ F68 or Poloxamer 188 (P188) was a free sample from BASF (Ludwigshafen, Germany). Cetyltrimethylammonium bromide (CTAB) and uranyl acetate were acquired from Sigma-Aldrich (Sintra, Portugal). Anhydrous glycerol was purchased from Acopharma (Barcelona, Spain). Ultra-purified water was obtained from a MiliQ Plus system (Milipore, Germany). All reagents were used without further treatment. The Y-79 human retinoblastoma cell line was purchased from Cell Lines Service (CLS, Eppelheim, Germany). Reagents for cell culture were from Gibco (Alfagene, Invitrogene, Portugal).
Table 1

Initial 3-level full factorial design, providing the lower ( -1$)$, medium ( 0$)$ and upper $(+1)$ level values for each variable.

\begin{tabular}{llll}
\hline Variables & Levels & & \\
\cline { 2 - 4 } & $\begin{array}{l}\text { Low level } \\
(-1)\end{array}$ & $\begin{array}{l}\text { Medium } \\
\text { level }(0)\end{array}$ & $\begin{array}{l}\text { High level } \\
(+1)\end{array}$ \\
\hline S100 (wt\%) & 2.5 & 5.0 & 7.5 \\
Lecithin (wt\%) & 0.25 & 0.5 & 0.75 \\
P188 (wt\%) & 0.5 & 1.0 & 1.0 \\
\hline
\end{tabular}

S100: Softisan ${ }^{\circledR} 100 ;$ P188: Poloxamer 188.

\subsection{Experimental factorial design}

A factorial design approach using a $3^{3}$ full factorial design composed of 3 variables which were set at 3-levels each was applied to maximize the experimental efficiency requiring a minimum of experiments. For this purpose three different variables and their influence on the physicochemical properties of the produced LN were analyzed. The design required a total of 11 experiments. The independent variables were the concentration of solid lipid S100, concentration of lecithin (Lipoid ${ }^{\circledR}$ S75) and the concentration of hydrophilic surfactant P188. The established dependent variables were the mean particle size (Z-Ave), polydispersity index (PI) and zeta potential (ZP). For each factor, the lower, medium and higher values of the lower, medium and upper levels were represented by $\mathrm{a}(-1), \mathrm{a}(0)$ and a (+1) sign, respectively (Table 1$)$. The data were analyzed using the STATISTICA 7.0 (Stafsoft, Inc.) software.

\subsection{Lipid nanoparticles production}

LN dispersions were prepared using a novel multiple emulsion (w/o/w) technique (García-Fuentes et al., 2003). Briefly, an inner w/o emulsion was initially prepared. A volume of ultra-purified water was added to the lipid phase (5 wt\%) composed of glycerol, S100 and Lipoid ${ }^{\circledR}$ S75 at same temperature $\left(5-10^{\circ} \mathrm{C}\right.$ above the melting point of the solid lipid Softisan ${ }^{\circledR} 100\left(T \approx 50^{\circ} \mathrm{C}\right)$ and homogenized $60 \mathrm{~s}$ with a sonication probe ( $6 \mathrm{~mm}$ diameter) by means of an Ultrasonic processor VCX500 (Sonics, Switzerland). A power output with amplitude of $40 \%$ was applied. A few milliliters of P188 solution was added and homogenized for additional $90 \mathrm{~s}$. This preemulsion was poured in the total volume of a P188 cooled solution under magnetic stirring for $15 \mathrm{~min}$ to allow the formation of the $\mathrm{LN}$. The obtained LN dispersions were used for subsequent studies. The general composition of LN dispersions is described in Table 2.

\subsection{Physicochemical characterization}

Physicochemical parameters such as Z-Ave, PI and ZP were analyzed by dynamic light scattering (DLS, Zetasizer Nano ZS, Malvern Instruments, Malvern, UK). All samples were diluted with ultrapurified water and analyzed in triplicate. For analysis of the ZP, ultra-purified water with conductivity adjusted to $-50 \mu \mathrm{S} / \mathrm{cm}$ was used.

Table 2

Composition of SLN dispersions (wt/wt\%).

\begin{tabular}{lc}
\hline Components & $\%(\mathrm{wt} / \mathrm{wt})$ \\
\hline Softisan $^{\circledR} 100$ & 5.0 \\
Glycerol $^{\circledR}$ & 37.5 \\
Lipoid $^{\circledR}$ S75 & 0.5 \\
Lutrol $^{\circledR}$ F68 & 1.0 \\
Water add. & 100 \\
\hline
\end{tabular}




\subsection{Evaluation of the concentration of CTAB}

In order to increase eye retention time and mucoadhesion onto the ocular mucosa, a cationic lipid was added to the lipid matrix. For this purpose, CTAB was used as cationic lipid maintaining the $5 \%$ of lipid matrix varying the proportions of S100 and CTAB. Thus, in the production stage, four different concentrations $(0.25,0.5,0.75$ and $1.0 \mathrm{wt} \%$ ) of cationic lipid (CTAB) was added in the lipid phase composed of S100 and Lipoid ${ }^{\circledR}$ S75 of the optimal formulation, previously obtained from the factorial design to evaluate the influence in the physicochemical properties.

\subsection{Stability analysis of $L N$ by TurbiscanLab ${ }^{\circledR}$}

The TurbiscanLab ${ }^{\circledR}$ is a technique used to observe reversible (creaming and sedimentation due to fluctuation on particle size and volume) and irreversible (coalescence and segregation due to particle size variation) destabilization phenomena in the sample without the need of dilution. TurbiscanLab ${ }^{\circledR}$ is useful to detect destabilization phenomena much earlier and also in a simpler way than other methods, since it is based on the measurement of backscattering (BS) and transmission (T) signals (Araújo et al., 2009; Celia et al., 2009; Marianecci et al., 2010; Liu et al., 2011).

The physical stability of LN dispersions was assessed with an optical analyzer TurbiscanLab ${ }^{\circledR}$ (Formulaction, France). The dispersions were placed in a cylindrical glass cell, at room temperature $\left(25^{\circ} \mathrm{C}\right)$. The equipment is composed of a near-infrared light source $(\lambda=880 \mathrm{~nm})$, and 2 synchronous transmission $(\mathrm{T})$ and backscattering (BS) detectors. The $\mathrm{T}$ detector receives the light crossing the sample, whereas the BS detector receives the light scattered backwards by the sample (Araújo et al., 2009). The detection head scans the entire height of the sample cell (20 mm longitude), acquiring $\mathrm{T}$ and BS each $40 \mu \mathrm{m}, 3$ times during $10 \mathrm{~min}$ at different times after production ( 7,15 and 30 days).

\subsection{Thermal analysis}

The crystallinity profile of LN was assessed by differential scanning calorimetry (DSC). This technique is useful to evaluate the physical state, which directly affects the physicochemical properties and thermodynamic stability of LN dispersions. A volume of LN dispersion corresponding to 1-2 mg of lipid was scanned using a Mettler DSC 823e System (Mettler Toledo, Spain). Heating and cooling runs were performed from $25^{\circ} \mathrm{C}$ to $90^{\circ} \mathrm{C}$ and back to $25^{\circ} \mathrm{C}$ at a heating rate of $5^{\circ} \mathrm{C} / \mathrm{min}$, in sealed $40 \mu \mathrm{L}$ aluminum pans. An empty pan was used as a reference. Indium (purity >99.95\%; Fluka, Buchs, Switzerland) was employed for calibration purposes. DSC thermograms were recorded for the four different CTAB concentration formulations and for the bulk lipids (CTAB and S100). The DSC parameters including onset, melting point and enthalpy were evaluated using STAR ${ }^{\mathrm{e}}$ Software (Mettler Toledo, Switzerland).

\subsection{X-Ray studies}

X-ray diffraction patterns were obtained using the X-ray scattering (X'Pert PRO, PANalytical) using a X'Celerator as a detector. Data of the scattered radiation were detected with a blend localsensible detector using an anode voltage of $40 \mathrm{kV}$ and a current of $30 \mathrm{~mA}$. For the analysis of LN dispersions and bulk materials, the samples were mounted on a standard sample holder being dried at room temperature without any previous sample treatment.

\subsection{Alamar blue assay in human retinoblastoma cell line}

Y-79 (Human retinoblastoma cell line) cells were used to perform the cytotoxicity assay, in which four LN dispersions containing different $\mathrm{CTAB}$ concentrations were tested. Each formulation was tested at four concentrations (in $\mu \mathrm{g} \mathrm{mL}^{-1}$ ): $10,25,50$ and 100. Y-79 cells were maintained in RPMI-1640, supplemented with $10 \%(\mathrm{v} / \mathrm{v})$ fetal bovine serum (FBS), $2 \mathrm{mM}$ L-glutamine, and antibiotics ( $100 \mathrm{U} \mathrm{mL}^{-1}$ penicillin and $100 \mu \mathrm{g} \mathrm{mL}^{-1}$ of streptomycin) in an atmosphere of $5 \% \mathrm{CO}_{2}$ in air at $37^{\circ}$ Cells were centrifuged, resuspended in FBS-free culture media, counted and seeded, after appropriate dilution, at $1 \times 10^{5}$ cell $\mathrm{mL}^{-1}$ density in 96 -well plates (100 $\mu \mathrm{L} /$ well). The different formulations were diluted with FBSfree culture media to achieve the final concentrations, and added to cells $24 \mathrm{~h}$ after seeding $(100 \mu \mathrm{L} /$ well $)$. Cell viability was assayed with Alamar Blue (Alfagene, Invitrogene, Portugal) by adding $10 \%$ $(\mathrm{v} / \mathrm{v})$ to each well, and the absorbance at $570 \mathrm{~nm}$ (reduced form) and $620 \mathrm{~nm}$ (oxidative form) was read 24,48 and $72 \mathrm{~h}$ after exposure to test compounds, data were analyzed by calculating the percentage of Alamar blue reduction (according to the manufactures recommendation) and expressed as percentage of control (untreated cells).

\subsection{Transmission electronic microscopy analysis}

Transmission electronic microscopy (TEM) is a technique useful to analyze the shape and size of LN dispersions. The chosen $\mathrm{LN}$ dispersion corresponding to CTAB-LN dispersion with $0.5 \mathrm{wt} \% \mathrm{CTAB}$ was mounted on a grid and negative stained with a $2 \%(\mathrm{v} / \mathrm{v})$ uranyl acetate solution. After drying at room temperature, the sample was examined using a TEM (Tecnai Spirit TEM, FEI) at $80 \mathrm{kV}$.

\subsection{Statistical analysis}

Statistical evaluation of data was performed using one-way analysis of variance (ANOVA). The Bonferroni multiple comparison test was used to compare the significance of the difference between the groups, a $p$-value $<0.05$ was accepted as significant. Data were expressed as the mean value \pm standard deviation (Mean \pm SD) $(n=3)$.

\section{Results and discussion}

The production and optimization of LN based on multiple emulsion requires pre-formulation studies and literature research. The stability and compatibility of the emulsifiers and the lipid matrix are essential to provide a stable and functional system (Fangueiro et al., 2012). Since the choice of the components are vital for dispersions formation, Lipoid ${ }^{\circledR}$ S75 (soybean phosphatidylcholine) was the selected lipophilic emulsifier used with a HLB value of approximately 7-9, and Poloxamer 188 was selected as hydrophilic emulsifier with a HLB value of approximately 22 .

Physicochemical properties and stability of the new drug delivery systems are major issues to be considered in the formulation stage, especially those intended for ocular administration. The use of dispersions with appropriate physicochemical properties ensures adequate bioavailability of administered drugs and biocompatibility with ocular mucosa. The LN dispersions obtained from the factorial design, revealed adequate physicochemical stability during the analytical testing. In addition, the macroscopic stability of the particles, monitored by visual analysis, DLS and Turbiscan $^{\circledR}$ Lab analysis, did not suffer any changes. Separation phase, flocculation or creaming phenomena were not reported in this study. The physicochemical properties of LN obtained from the experimental design were analyzed. The effects of the selected variables on dispersions characteristics (Z-Ave, PI and ZP) are depicted in Table 3. For drug ocular administration, the values of $Z$-Ave and PI should be the lowest as possible, since dispersions should be well tolerated in the ocular mucosa avoiding eye irritation and the transport and uptake from the cornea is facilitated (Araujo et al., 2009). 
Table 3

Response values (Z-Ave, PI and ZP) of the three factors depicted in Table 1 for the 11 experiment formulations.

\begin{tabular}{|c|c|c|c|c|c|c|}
\hline Run & S100 (wt\%) & Lecithin (wt\%) & P188 (wt\%) & Z-Ave $(\mathrm{nm}) \pm \mathrm{SD}$ & $\mathrm{PI} \pm \mathrm{SD}$ & $\mathrm{ZP}(\mathrm{mV})$ \\
\hline SLN1 & 2.5 & 0.25 & 0.5 & $256.40 \pm 1.20$ & $0.181 \pm 0.02$ & -1.04 \\
\hline SLN2 & 7.5 & 0.25 & 0.5 & $268.50 \pm 1.47$ & $0.173 \pm 0.04$ & -1.06 \\
\hline SLN3 & 2.5 & 0.75 & 0.5 & $165.85 \pm 2.21$ & $0.162 \pm 0.01$ & -1.08 \\
\hline SLN4 & 7.5 & 0.75 & 0.5 & $171.24 \pm 1.78$ & $0.155 \pm 0.03$ & -1.20 \\
\hline SLN5 & 2.5 & 0.25 & 1.5 & $241.30 \pm 2.54$ & $0.182 \pm 0.05$ & -1.22 \\
\hline SLN6 & 7.5 & 0.25 & 1.5 & $235.40 \pm 1.87$ & $0.192 \pm 0.02$ & -1.04 \\
\hline SLN7 & 2.5 & 0.75 & 1.5 & $189.75 \pm 1.99$ & $0.163 \pm 0.08$ & -1.12 \\
\hline SLN8 & 7.5 & 0.75 & 1.5 & $164.50 \pm 1.14$ & $0.158 \pm 0.03$ & -1.14 \\
\hline SLN9 & 5.0 & 0.5 & 1.0 & $165.90 \pm 1.20$ & $0.164 \pm 0.04$ & -1.18 \\
\hline SLN10 & 5.0 & 0.5 & 1.0 & $164.50 \pm 1.09$ & $0.183 \pm 0.05$ & -1.17 \\
\hline SLN11 & 5.0 & 0.5 & 1.0 & $164.70 \pm 1.01$ & $0.177 \pm 0.02$ & -1.16 \\
\hline
\end{tabular}

Table 4

ANOVA statistical analysis of the Z-Ave.

\begin{tabular}{|c|c|c|c|c|c|}
\hline Evaluated factors and their interactions & Sum of squares & Degrees of freedom & Mean square & F-value & $p$-Value \\
\hline (1) S100 concentration & 23.32 & 1 & 23.32 & 0.01962 & 0.895380 \\
\hline (2) Lecithin concentration & 12032.66 & 1 & 12032.66 & 10.12031 & 0.033495 \\
\hline (3) P188 concentration & 120.44 & 1 & 120.44 & 0.10129 & 0.766206 \\
\hline 1 by 2 & 84.89 & 1 & 84.89 & 0.07140 & 0.802522 \\
\hline 1 by 3 & 295.73 & 1 & 295.73 & 0.24873 & 0.644149 \\
\hline 2 by 3 & 533.99 & 1 & 533.99 & 0.44912 & 0.539455 \\
\hline Error & 4755.85 & 4 & 1188.96 & & \\
\hline Total & 17846.88 & 10 & & & \\
\hline
\end{tabular}

The values in bold are the statiscally significant results $(p<0.5)$.

Table 5

ANOVA statistical analysis of the PI.

\begin{tabular}{|c|c|c|c|c|c|}
\hline Evaluated factors and their interactions & Sum of Squares & Degrees of freedom & Mean square & $F$-value & $p$-Value \\
\hline (1) S100 concentration & 0.000072 & 1 & 0.000072 & 1.02591 & 0.368411 \\
\hline (2) Lecithin concentration & 0.001352 & 1 & 0.001352 & 19.26425 & 0.011791 \\
\hline (3) P188 concentration & 0.000032 & 1 & 0.000032 & 0.45596 & 0.536541 \\
\hline 1 by 2 & 0.000032 & 1 & 0.000032 & 0.45596 & 0.536541 \\
\hline 1 by 3 & 0.000072 & 1 & 0.000072 & 1.02591 & 0.368411 \\
\hline 2 by 3 & 0.000032 & 1 & 0.000032 & 0.45596 & 0.536541 \\
\hline Error & 0.000281 & 4 & 0.000070 & & \\
\hline Total & 0.001873 & 10 & & & \\
\hline
\end{tabular}

The values in bold are the statiscally significant results $(p<0.5)$.

The results for the 11 produced formulations is shown in Table 3 and varied from $164.5 \pm 1.09 \mathrm{~nm}$ (LN 8 or LN 10) to $268.50 \pm 1.47 \mathrm{~nm}$ (LN 2), whereas PI ranged from $0.155 \pm 0.03$ (LN 4) to $0.192 \pm 0.02$ (LN 6). The particle size distribution was very narrow in all cases since the PI was less than 0.2, corresponding to monodispersed systems. According to the literature (Zimmer and Kreuter, 1995, Shekunov et al., 2007), the Z-ave for ocular administration should be below $1 \mu \mathrm{m}$ with an associated narrow size distribution. Thus, all formulations revealed a Z-Ave and PI within accepted range (Fresta et al., 1999; Giannavola et al., 2003; Vega et al., 2008; Souto et al., 2010). As expected, the ZP did not vary, since all used reagents have non-ionic nature. For each of the 3 variables, analysis of variance (ANOVA) was performed. From Table 4 and Fig. 1, the only factor that was shown to have a significant effect ( $p$-value $<0.05$ ) on Z-Ave was the concentration of lecithin. All other evaluated factors, were not statistically significant ( $p$-value $>0.05$ ), neither the interactions between them. The same results were observed for the evaluation of the dependent variables on PI (Table 5 and Fig. 1). The
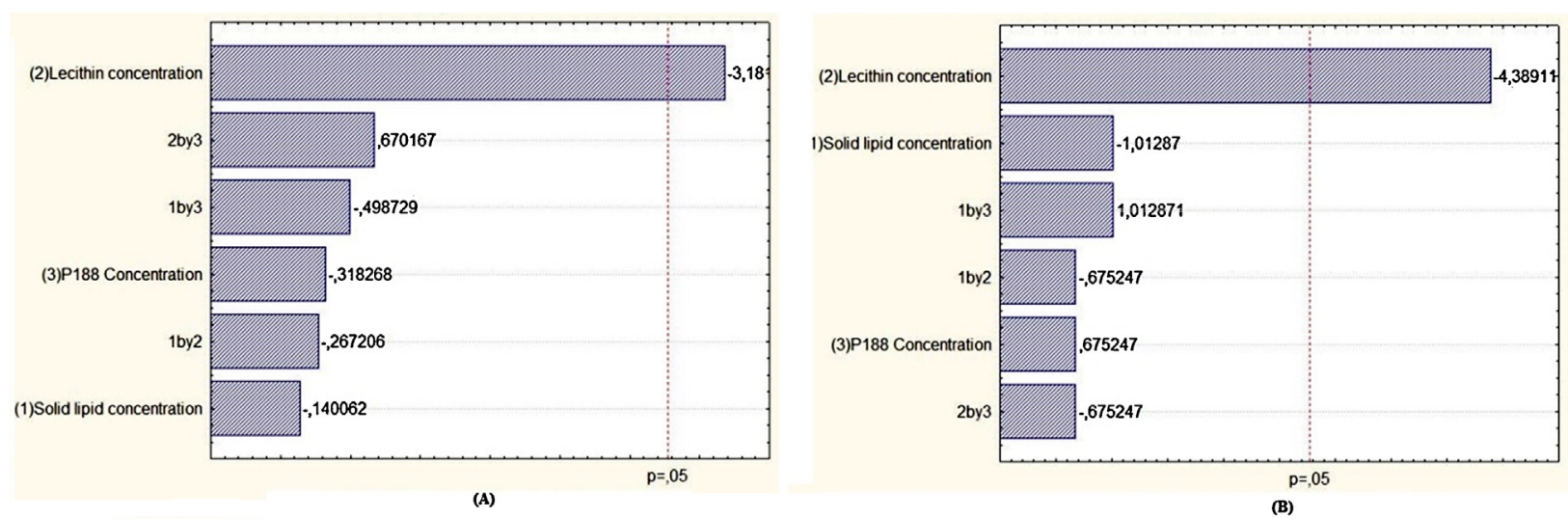

Fig. 1. Pareto chart of the analyzed effects for the Z-Ave (A) and for the PI (B). 

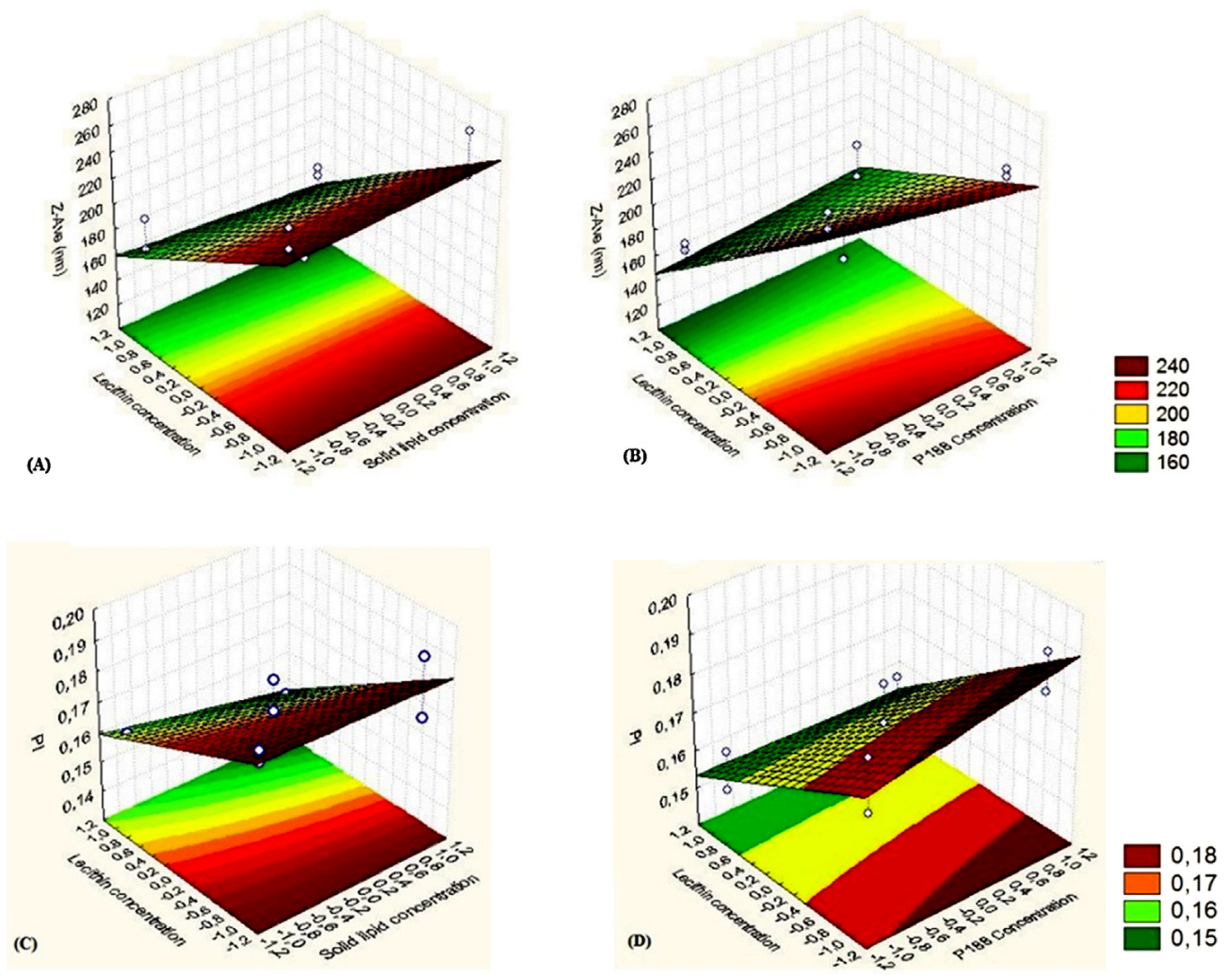

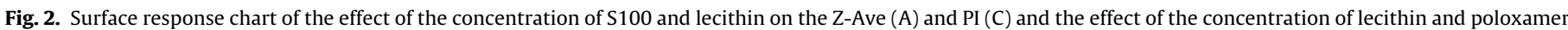
188 on the Z-Ave (B) and PI (D).

concentration of lecithin was the only independent variable affecting the PI. The lecithin used is composed of $75 \%$ of phosphatidylcholine, which is composed by phospholipids that resemble the cellular membranes. The use of this emulsifier is reported as safe and biocompatible for several purposes, such as skin products (Fiume, 2001) and also for ophthalmic/ocular drug delivery (Bhatta et al., 2012). Their use in the development of LN dispersions is essential to decrease the interfacial tension between the oil phase and the internal and external aqueous phase, and also to facilitate the emulsification of the lipid matrix. Lecithin is used due to its higher power of emulsification able to provide a very good stabilization of the oil-in-water interfaces and has also been reported to decrease particle size in emulsions that is mainly explained by its amphiphilic character (Trotta et al., 2002; Schubert et al., 2006; Kawaguchi et al., 2008). The lipophilic portion of lecithin dissolves the lipid phase, i.e. lecithin likes to be at the edge of the lipid phase being its lipophilic tails directed to the lipid phase until the hydrophilic portion is directed to the water phase. Thus, the oil phase is totally recovered by the lecithin promoting long time stabilization in the interface of the emulsions (Trotta et al., 2002). From the obtained results, a correlation between the concentration of lecithin and mean particle size of the particles were found, since highest concentrations leads to a decrease in Z-Ave and PI. This dependency of Z-Ave on the type of emulsifier is due the need for the complete coverage of the interface, which is affected by the selected concentration (Fig. 2).

The aim of this factorial design was to optimize a formulation with appropriate physicochemical parameters for the incorporation of hydrophilic drugs for ocular delivery. For this purpose, the limiting factors were the Z-Ave and PI and from the obtained results an optimal LN dispersion was found. This LN dispersion was used for the following studies.

In order to improve LN adhesion to ocular surface and also to improve stability of the dispersions, a cationic lipid was used. The approach of using a cationic lipid is interesting since ocular mucosa depicts slightly negative charge above its isoelectric point and also could improve some limitations related to ocular administration, such as prevent tear washout (due to tear dynamics), increase ocular bioavailability and prolong the residence time of drugs in the cul-de-sac (Araujo et al., 2009).

The study reports the use of different concentrations of a cationic lipid $(C T A B)$ in the lipid matrix of LN. The results of the CTAB concentration on the Z-Ave, PI and ZP of the LN dispersions optimized in the factorial design are depicted in Table 6. As expected, the parameter most affected by the variation on CTAB concentration is the ZP. The increase of the ZP was directly proportional to the increase on $C T A B$ concentration. Statistical analysis of the physicochemical properties of CTAB-LN dispersions was not significant $(p>0.05)$, indicating that the concentration of CTAB did not affect drastically the Z-Ave, PI and ZP of the formulations. All formulations properties were in agreement with the required parameters for ocular delivery. However, no statistical differences were found, the concentration of CTAB improved the ZP following a proportional relationship. This behavior was expected because of the cationic properties of CTAB and is also expected that higher ZP values contribute to higher stability of the particles due to electronic repulsion between them maintaining longer time in suspension.

The BS signal is graphically reported as positive (BS increase) or negative peak (BS decrease). The migration of particles to the top of the cell leads to a concentration decrease at the bottom, translated by a decrease in the BS signal (negative peak) and vice versa for the phenomena occurred on the top of the cell. If the BS profiles have a deviation of $\leq \pm 2 \%$ it can be considered that there are no significant variations in particle size. Variations up to $\pm 10 \%$ indicate unstable formulations (Araújo et al., 2009; Celia et al., 2009). From the results depicted in Fig. 3 it is possible to detect instability due the variation up $10 \%$ on the CTAB-LN composed of $0.25 \mathrm{wt} \%$ of CTAB, indicating that this concentration is not sufficient to promote LN dispersion 
Table 6

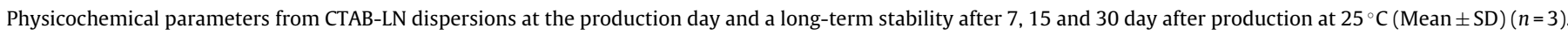

\begin{tabular}{|c|c|c|c|c|c|}
\hline Formulation & Parameters & Production day & Day 7 & Day 15 & Day 30 \\
\hline $0.25 \%$ CTAB-LN & $\begin{array}{l}\text { Z-Ave }(\mathrm{nm}) \\
\text { PI } \\
\mathrm{ZP}(\mathrm{mV})\end{array}$ & $\begin{aligned} 230.70 & \pm 6.71 \\
0.308 & \pm 0.09 \\
+24.80 & \pm 2.69\end{aligned}$ & $\begin{array}{l}239.5 \pm 0.61 \\
0.267 \pm 0.01 \\
+17.4 \pm 0.65\end{array}$ & $\begin{array}{l}244.9 \pm 1.17 \\
0.261 \pm 0.03 \\
+16.7 \pm 0.56\end{array}$ & $\begin{array}{l}255.2 \pm 1.32 \\
0.266 \pm 0.01 \\
+16.4 \pm 0.36\end{array}$ \\
\hline $0.5 \%$ CTAB-LN & $\begin{array}{l}\text { Z-Ave }(\mathrm{nm}) \\
\text { PI } \\
\mathrm{ZP}(\mathrm{mV})\end{array}$ & $\begin{aligned} 194.40 & \pm 0.43 \\
0.185 & \pm 0.02 \\
+37.20 & \pm 1.27\end{aligned}$ & $\begin{aligned} 199.25 & \pm 0.30 \\
0.186 & \pm 0.01 \\
+33.8 & \pm 0.45\end{aligned}$ & $\begin{aligned} 201.45 & \pm 0.08 \\
0.224 & \pm 0.02 \\
+32.1 & \pm 0.53\end{aligned}$ & $\begin{array}{l}213.7 \pm 0.74 \\
0.225 \pm 0.01 \\
+30.5 \pm 0.03\end{array}$ \\
\hline $0.75 \%$ CTAB-LN & $\begin{array}{l}\text { Z-Ave }(\mathrm{nm}) \\
\text { PI } \\
\mathrm{ZP}(\mathrm{mV})\end{array}$ & $\begin{aligned} 172.10 & \pm 12.64 \\
0.182 & \pm 0.02 \\
+41.70 & \pm 0.71\end{aligned}$ & $\begin{aligned} 166.25 & \pm 1.20 \\
0.246 & \pm 0.01 \\
+40.15 & \pm 0.63\end{aligned}$ & $\begin{array}{r}223 \pm 0.59 \\
0.204 \pm 0.01 \\
+38.6 \pm 0.41\end{array}$ & $\begin{array}{l}236.8 \pm 1.45 \\
0.225 \pm 0.04 \\
+37.4 \pm 1.21\end{array}$ \\
\hline $1.0 \%$ CTAB-LN & $\begin{array}{l}\text { Z-Ave }(\mathrm{nm}) \\
\text { PI } \\
\mathrm{ZP}(\mathrm{mV})\end{array}$ & $\begin{aligned} 169.10 & \pm 2.51 \\
0.222 & \pm 0.022 \\
+48.00 & \pm 0.31\end{aligned}$ & $\begin{aligned} 144.7 & \pm 1.61 \\
0.211 & \pm 0.01 \\
+44.58 & \pm 0.50\end{aligned}$ & $\begin{array}{l}188.3 \pm 1.52 \\
0.236 \pm 0.02 \\
+42.0 \pm 0.05\end{array}$ & $\begin{array}{l}200.7 \pm 1.65 \\
0.245 \pm 0.04 \\
+40.2 \pm 0.63\end{array}$ \\
\hline
\end{tabular}

stability over time. The other concentrations seem to be more stable since variations are lower than $10 \%$ during the time of analysis. This analysis could predict the good stability of the formulations with CTAB concentration ranging between 0.5 and $1.0 \mathrm{wt} \%$. These results are in agreement with the $\mathrm{ZP}$ values recorded for the four formulations (Table 6), since higher ZP values could also predict a higher stability of LN dispersions as mentioned before. In order to support Turbiscan ${ }^{\circledR}$ Lab results and also to monitored particles over a period of time, zetasizer measurements were made at the same days after CTAB-LN dispersions production during storage at $25^{\circ} \mathrm{C}$. All dispersions showed a milky colloidal appearance where no aggregation phenomena and no phase separation were detected during the period of analysis and storage. From the results depicted in Table 6 is possible to detect a slightly increase both in the Z-Ave and in the PI after 30 days of storage. During the period of analysis, CTAB-LN dispersions depicted particle sizes below $300 \mathrm{~nm}$ and $\mathrm{PI} \leq 0.3$, which are acceptable values for ocular delivery (Vega et al., 2008; Gonzalez-Mira et al., 2010; Souto et al., 2010; Gonzalez-Mira et al., 2011). The long-term stability studies confirmed that concentrations of CTAB up to $0.5 \mathrm{wt} \%$ could provide a better electrostatic repulsion between particles in suspension and from this suggestion provide better stability avoiding particle aggregation and/or flocculation.

The analysis of the cristallinity and polymorphism of the LN dispersions obtained was analyzed by DSC and X-Ray. The thermodynamic stability of LN depends mainly on the lipid modification (a)

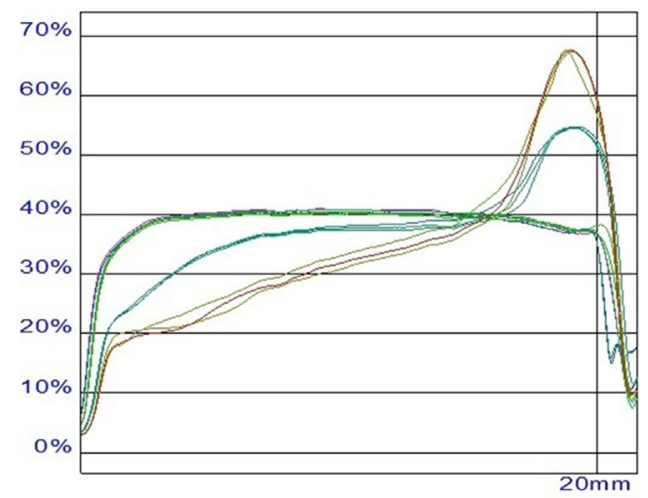

(c)

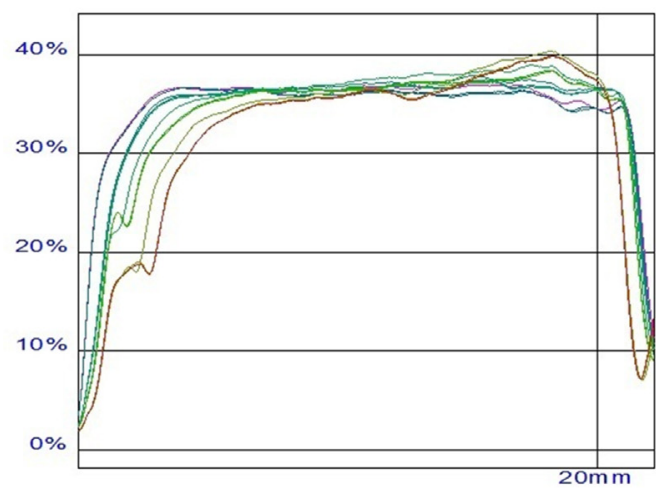

Production day (b)

(d)
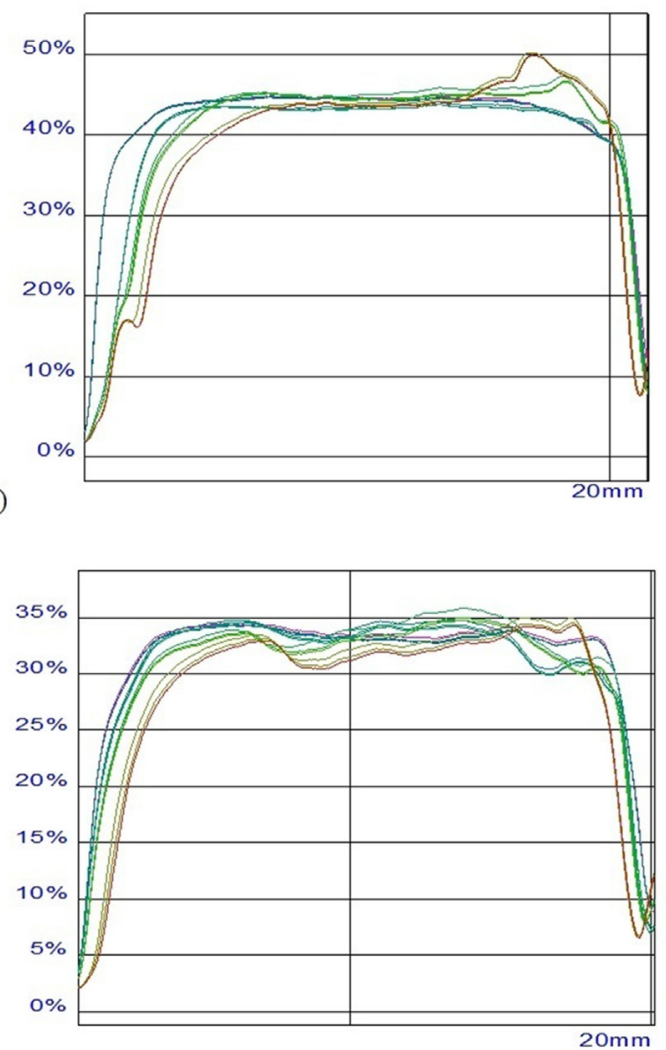

Day 15

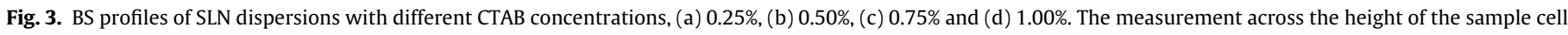
during $10 \mathrm{~min}$, on the production day, day 7 , day 15 and day 30 after the production $(n=3)$. 


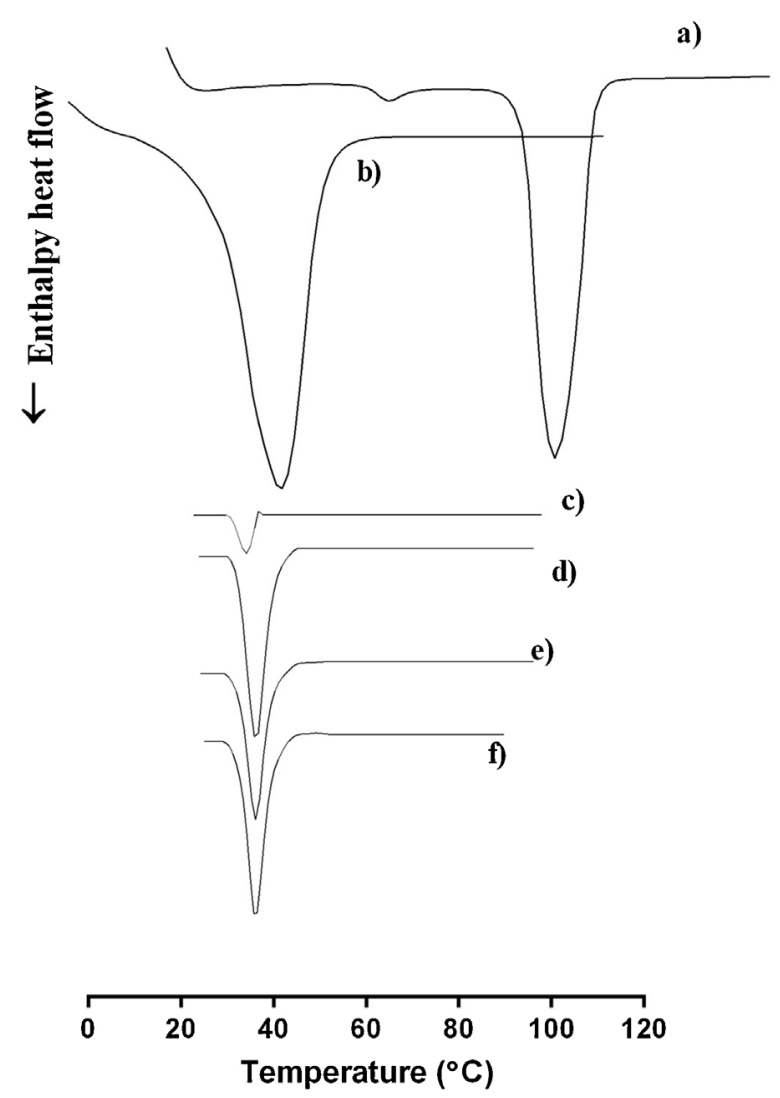

Fig. 4. DSC thermograms of (a) bulk CTAB, (b) bulk S100 and CTAB-LN with different concentrations (c) $0.25 \mathrm{wt} \%$, (d) $0.50 \mathrm{wt} \%$, (e) $0.75 \mathrm{wt} \%$ and (f) $1.0 \mathrm{wt} \%$.

that occurs after crystallization. The solid lipid used was S100, a triacylglycerol blend of vegetable fatty acids with $\mathrm{C}_{10}-\mathrm{C}_{18}$ (Fangueiro et al., 2013). Polymorphic transitions after crystallization of triacylglycerol based LN are slower for longer-chain triacylglycerols than for shorter-chain triacylglycerol (Metin and Hartel, 2005). The type of emulsifier used in LN dispersions also affects their thermodynamic behavior, their storage time and degradation velocity (Han et al., 2008).

Triacylglycerols usually occur in three major polymorphic forms, namely $a, \beta^{\prime}$ and $\beta$ (in order of increasing thermodynamic stability) which are characterized by different sub cell packing of the lipid chains. The $\beta^{\prime}$ modification is frequently observed in complex triacylglycerols such as S100 (Bunjes and Unruh, 2007).

Thermal analysis of the bulk lipid S100 (Fig. 4) shows a single endothermic peak upon heating with a minimum of $39.12^{\circ} \mathrm{C}$ and an enthalpy of $-44.76 \mathrm{Jg}^{-1}$ (Table 7 ). Our results are in agreement with those reported by other authors (Thoma and Serno, 1983; Schubert and Müller-Goymann, 2003) for a $\beta^{\prime}$ modification of complex triacylglycerols mixtures. For CTAB bulk, a small melting event was observed between 40 and $50^{\circ} \mathrm{C}$, that can be associated to the melting of acyl chains and a main transition at around $106.34^{\circ} \mathrm{C}$ corresponding to complete melting of CTAB and attributed to the melting of head-groups (Doktorovova et al., 2011).

With respect to CTAB-LN dispersions (Fig. 4), a very small endothermic event was reported compared to the other thermograms of the bulk lipid and bulk matrix. This transition is relatively small due to the lowest values of enthalpy presented by the LN dispersions compared to the bulk lipid (Table 7). The peak temperature slightly decreased for all CTAB-LN dispersions confirming that polymorphism of S100. In LN dispersions, a slight decrease of enthalpy is usually observed, as well as a decrease on the peak temperature comparing to the bulk counterpart, however since this lipid has a

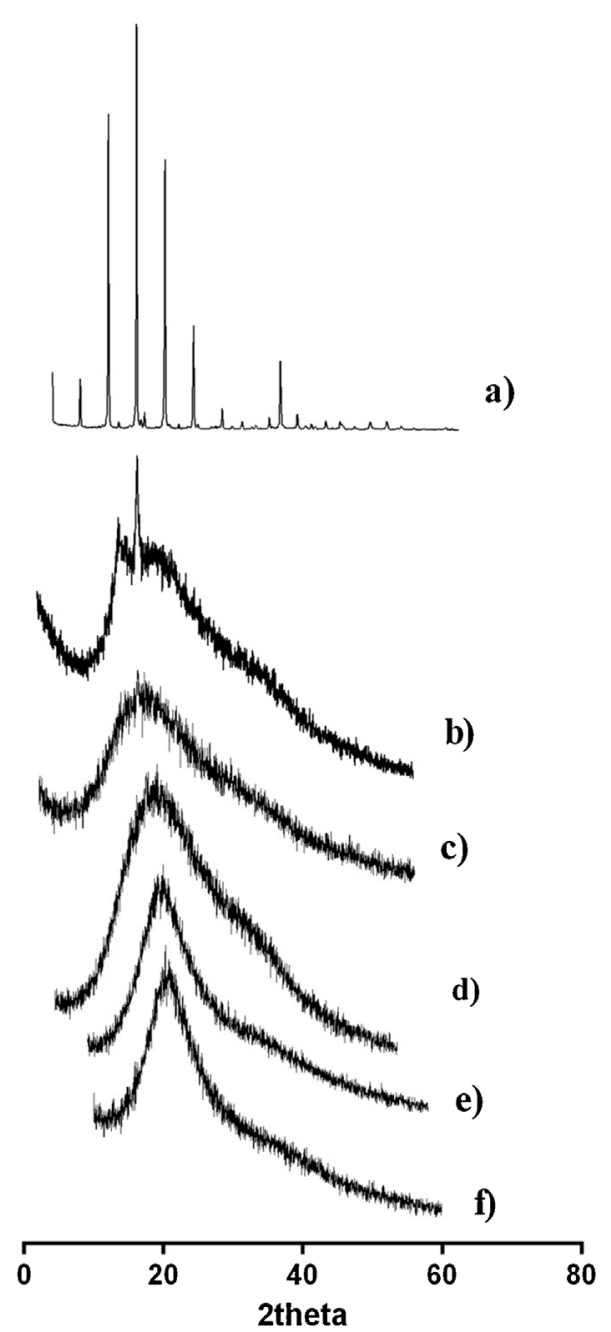

Fig. 5. X-ray diffraction patterns of (a) bulk CTAB, (b) bulk S100 and CTAB-LN with different concentrations (c) $0.25 \mathrm{wt} \%$, (d) $0.50 \mathrm{wt} \%$, (e) $0.75 \mathrm{wt} \%$ and (f) $1.0 \mathrm{wt} \%$.

very low melting point, it is possible to form supercooled melts. A possible explanation for the reduction of crystallinity of the $\mathrm{LN}$ dispersion is the coexistence of lipid being present in the $a$ modification and also due to the colloidal particle size offering insufficient number of diffraction levels. These differences can be attributed to the high surface to volume ratio of LN dispersions (Schubert and Müller-Goymann, 2003).

The X-ray results depicted in Fig. 5 also reveal the presence of two signals one at $0.42 \mathrm{~nm}\left(2 \theta=21.1^{\circ}\right)$ and other at $0.38 \mathrm{~nm}$ $\left(2 \theta=23.2^{\circ}\right)$ which are both characteristic of the orthorhombic perpendicular subcell, i.e., $\beta^{\prime}$ modification (Schubert and MüllerGoymann, 2003). These results are in agreement with DSC studies, since all formulations revealed a decreased crystallinity comparing with bulk lipids S100 and CTAB. Also, the melting enthalpy of CTAB-LN dispersions is increasing with increasing CTAB content, indicating higher crystallinity of the matrices containing $0.5-1.0$ wt\% of CTAB. Thus, the concentration of CTAB in the lipid matrix is able to provide higher crystallinity to the lipid matrix; however, other features such as stability and toxicity were analyzed to provide a full study in order to choose the best CTAB concentration.

LN toxicity is essential to design drug delivery systems able to ensure security and avoid side effects. Cytotoxicity was assessed in human retinoblastoma cell line, Y-79. The Alamar blue assay measures quantitatively the proliferation of the cells establishing cytoxicity of tested agents/drugs and can be used as a baseline 
Table 7

Differential scanning calorimetry (DSC) analysis of the SLN formulations with different CTAB concentrations.

\begin{tabular}{|c|c|c|c|c|}
\hline Formulation & Onset temperature $\left({ }^{\circ} \mathrm{C}\right)$ & Melting point $\left({ }^{\circ} \mathrm{C}\right)$ & Integral (mJ) & Enthalpy $\left(\mathrm{Jg}^{-1}\right)$ \\
\hline Softisan 100 bulk & 36.68 & 39.12 & -3111.15 & -44.76 \\
\hline CTAB bulk & 100.88 & 106.34 & -677.30 & -12.72 \\
\hline CTAB-LN 0.25 wt\% & 29.58 & 34.12 & -17.50 & -0.23 \\
\hline CTAB-LN 0.5 wt $\%$ & 31.26 & 35.03 & -116.43 & -1.47 \\
\hline CTAB-LN 0.75 wt $\%$ & 31.12 & 34.73 & -110.44 & -1.41 \\
\hline CTAB-LN 1.0 wt\% & 31.65 & 34.94 & -129.98 & -1.59 \\
\hline
\end{tabular}

for further in vivo studies. This assay could predict if formulations cause cellular damage which consequently results in loss of the metabolic cell function. Alamar blue is a sensitive fluorometric/colorimetric growth indicator used to detect metabolic activity of cells. Specifically, cells incorporate an oxidation-reduction (REDOX) indicator that, when in a reducing environment of 0 metabolically active cell, is reduced. When reduced, Alamar blue becomes fluorescent and changes color from blue to pink. The reduction of Alamar blue is believed to be mediated by mitochondrial enzymes (Hamid et al., 2004). However, some authors also suggest that cytosolic and microsomal enzymes also contribute to the reduction of Alamar blue (Gonzalez and Tarloff, 2001).

The evaluation of the Alamar blue assay was based on the percent viability of four concentrations for each CTAB concentration in the dispersion. This assay is important to point out the importance of the CTAB quantity in the formulation that could be administered for ocular delivery, avoiding cell damage. From Fig. 6, we can observe that $10 \mu \mathrm{g} \mathrm{mL}^{-1}$ of all CTAB-LN formulations is non-toxic to cells as cell viability is not statistically different from control (untreated cells) along the 3 time-points of exposure. The concentration of $50 \mu \mathrm{g} \mathrm{mL}^{-1}$ of CTAB-LN only reduced significantly cell viability in the CTAB-LN dispersions containing 0.75 and $1.0 \mathrm{wt} \%$ of $\mathrm{CTAB}$, along the 3 time-points of exposure. The concentration of $100 \mu \mathrm{g} \mathrm{mL}^{-1}$ of CTAB-LN reduced significantly cell viability in all CTAB percentages. CTAB-LN formulation containing $0.25 \%(\mathrm{w} / \mathrm{w})$ of CTAB is non-toxic for the range of $10-50 \mu \mathrm{g} \mathrm{mL}^{-1}$ (Fig. 6a), but reduces significantly cell viability, in about $50-75 \%$ of control $(p<0.05)$. The concentration range where cell viability is kept similar to control values is reduced increasing CTAB concentration in the formulations. In Fig. $6 \mathrm{~b}(0.5 \%$ of CTAB, concentration twice that used for Fig. 6a) we can observe that $50 \mu \mathrm{g} \mathrm{mL}^{-1}$ of formulation reduces cell viability from 40 to $60 \%$ of the control while $100 \mu \mathrm{g} \mathrm{mL}^{-1}$ practically abolishes cell viability for the 3 time points of exposure. Raising CTAB concentration in the formulation to $0.75 \%$ (Fig. 6c) and to $1.0 \%$ (Fig. $6 \mathrm{~d}$ ) reduces cell tolerance to the formulation as we observe that with increasing \% of CTAB in the formulation reduces the concentration that maintains cell viability. These results suggest that a higher CTAB concentration implies higher cytotoxicity which is expected since the effect of cationic agents on the human health is concentration dependent. In addition, higher CTAB-LN concentrations also imply more cytotoxicity. From our results, a safe and biocompatible LN system should be composed of, at maximum, $0.5 \mathrm{wt} \%$ of CTAB.

TEM analysis has been performed to evaluate the particle shape and morphology of CTAB-LN dispersion. TEM image (Fig. 7) shows a)

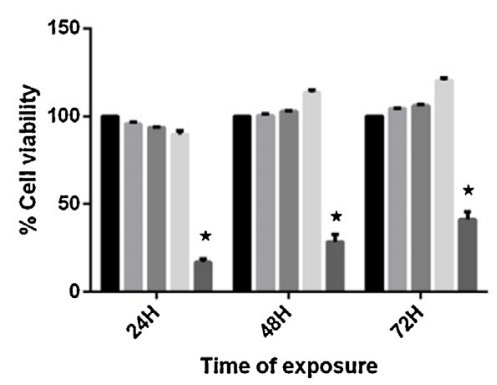

c)
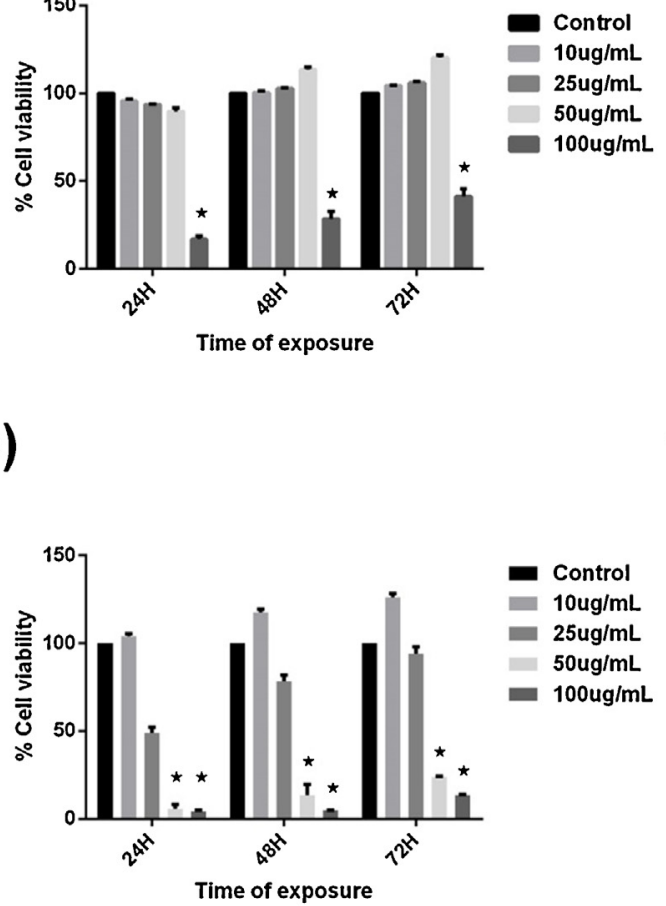

b)

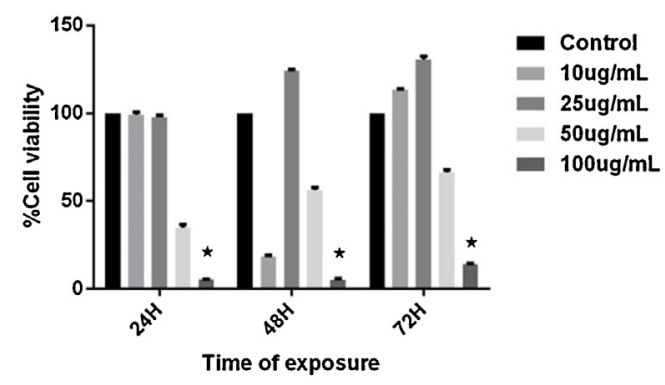

d)

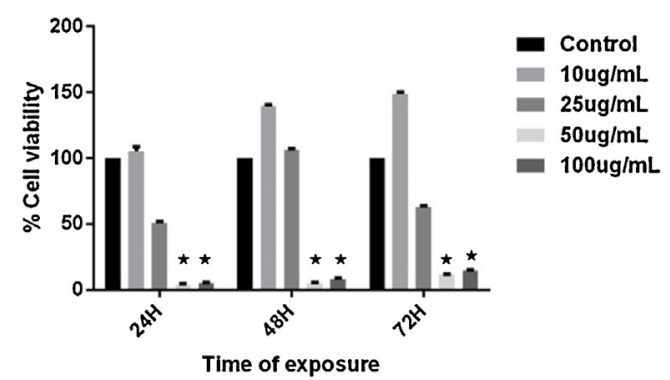

Fig. 6. Effect of CTAB-LN (a) $0.25 \%$; (b) $0.5 \%$; (c) $0.75 \%$ and d) $1.0 \%$ on Y-79 human retinoblastoma cells viability ( $n=4$ ) after 24,48 and 72 h of exposure, ${ }^{*} p<0.05$. 


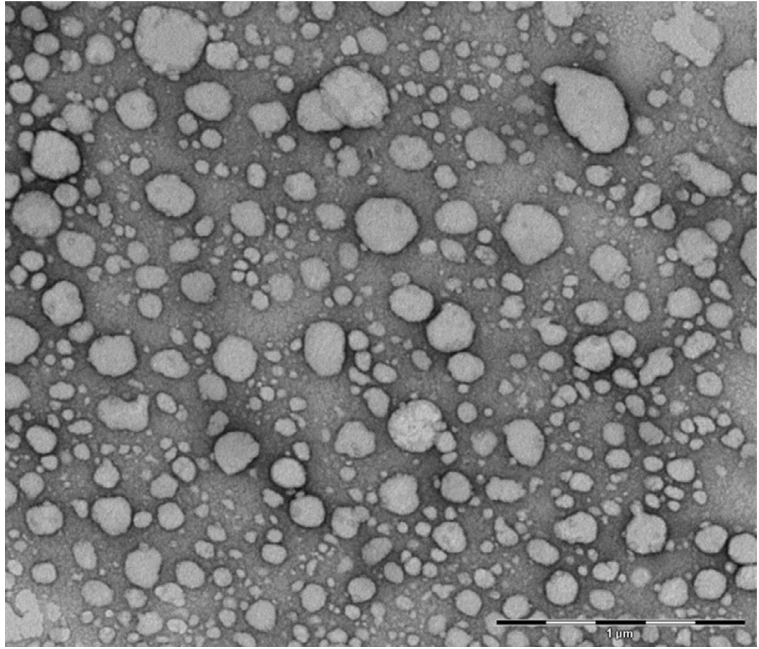

Fig. 7. TEM micrograph of $0.5 \%$ CTAB-LN.

particles mainly with spherical morphology. From this analysis, the absence of aggregation phenomena of CTAB-LN dispersion was also confirmed. These results are in agreement with Turbiscan ${ }^{\circledR} \mathrm{Lab}$ results. It is possible to detect a slight polydispersity however all the particles remain within the nanometer range. All particles showed a mean diameter that varied between 190 and $280 \mathrm{~nm}$, which is also in agreement with the zetasizer measurements. TEM analysis suggested that immediately after production CTAB-LN dispersion with $0.5 \mathrm{wt} \%$ CTAB contained particles no higher than $1 \mu \mathrm{m}$, which is useful for ocular delivery purposes.

\section{Conclusions}

The development of LN dispersions for ocular delivery should be carried out regarding ocular morphology and biology. A full factorial design was carried out in order to find out which parameters could influence LN dispersions based on multiple emulsion technique. The size and PI of LN dispersions are highly dependent on the lecithin concentration mainly due to its higher emulsification properties and amphiphilic character able to decrease particle size in emulsions. In order to improve ocular mucoadhesion, a cationic lipid was added in the lipid matrix. The insufficient information and lack of studies regarding nanotoxicity of cationic agents for ocular or drug delivery leads us to study different CTAB concentrations on lipid matrix and its effects on the physicochemical parameters and cell toxicity of CTAB-LN dispersions. This study demonstrated that the better $\mathrm{CTAB}$ concentration for the dispersion previously optimized by the factorial design was $0.5 \%$, providing better stability and biocompatibility. Further studies encapsulating hydrophilic drugs and evaluating the ex vivo and in vivo performance of the developed CTAB-LN dispersion are required to confirm these preliminary results.

\section{Acknowledgements}

Ms. Joana Fangueiro and Ms. Tatiana Andreani wish to acknowledge Fundação para a Ciência e Tecnologia do Ministério da Ciência e Tecnologia (FCT, Portugal) under the references SFRH/BD/80335/2011 and SFRH/BD/60640/2009, respectively. FCT is also acknowledged under the research project PTDC/SAU-FAR/113100/2009 and FCOMP-01-0124-FEDER-022696 (PEst-C/AGR/UI4033/2011).

\section{References}

Araujo, J., Gonzalez, E., Egea, M.A., Garcia, M.L., Souto, E.B., 2009. Nanomedicines for ocular NSAIDs: safety on drug delivery. Nanomedicine 5, 394-401.

Araújo, J., Vega, E., Lopes, C., Egea, M.A., Garcia, M.L., Souto, E.B., 2009. Effect of polymer viscosity on physicochemical properties and ocular tolerance of FBloaded PLGA nanospheres. Colloids Surf. B 72, 48-56.

Bhatta, R.S., Chandasana, H., Chhonker, Y.S., Rathi, C., Kumar, D., Mitra, K., Shukla, P.K., 2012. Mucoadhesive nanoparticles for prolonged ocular delivery of natamycin: in vitro and pharmacokinetics studies. Int. J. Pharm. 432, 105-112.

Bunjes, H., Unruh, T., 2007. Characterization of lipid nanoparticles by differentia scanning calorimetry, X-ray and neutron scattering. Adv. Drug Delivery Rev. 59 379-402.

Celia, C., Trapasso, E., Cosco, D., Paolino, D., Fresta, M., 2009. Turbiscan lab expert analysis of the stability of ethosomes and ultradeformable liposomes containing a bilayer fluidizing agent. Colloids Surf. B 72, 155-160.

del Pozo-Rodriguez, A., Delgado, D., Gascon, A.R., Solinis, M.A., 2013. Lipid nanoparticles as drug/gene delivery systems to the retina. J. Ocul. Pharmacol. Ther. 29, 173-188.

Diebold, Y., Calonge, M., 2010. Applications of nanoparticles in ophthalmology. Prog Retin. Eye Res. 29, 596-609.

Doktorovova, S., Shegokar, R., Rakovsky, E., Gonzalez-Mira, E., Lopes, C.M., Silva, A.M., Martins-Lopes, P., Muller, R.H., Souto, E.B., 2011. Cationic solid lipid nanoparticles (cSLN): structure, stability and DNA binding capacity correlation studies. Int. J. Pharm. 420, 341-349.

Fangueiro, J.F., Andreani, T., Egea, M.A., Garcia, M.L., Souto, S.B., Souto, E.B., 2012 Experimental factorial design applied to mucoadhesive lipid nanoparticles via multiple emulsion process. Colloids Surf. B 100, 84-89.

Fangueiro, J.F., Gonzalez-Mira, E., Martins-Lopes, P., Egea, M.A., Garcia, M.L., Souto, S.B., Souto, E.B., 2013. A novel lipid nanocarrier for insulin delivery: production, characterization and toxicity testing. Pharm. Dev. Technol. 18 545-549.

Fiume, Z., 2001. Final report on the safety assessment of lecithin and hydrogenated lecithin. Int. J. Toxicol. 20, 21-45.

Fresta, M., Panico, A.M., Bucolo, C., Giannavola, C., Puglisi, G., 1999. Characterization and in-vivo ocular absorption of liposome-encapsulated acyclovir. J. Pharm. Pharmacol. 51, 565-576.

Gallarate, M., Trotta, M., Battaglia, L., Chirio, D., 2009. Preparation of solid lipid nanoparticles from $\mathrm{W} / \mathrm{O} / \mathrm{W}$ emulsions: preliminary studies on insulin encapsulation. J. Microencapsul. 26, 394-402.

García-Fuentes, M., Torres, D., Alonso, M.J., 2003. Design of lipid nanoparticles for the oral delivery of hydrophilic macromolecules. Colloids Surf. B 27, 159-168.

Giannavola, C., Bucolo, C., Maltese, A., Paolino, D., Vandelli, M.A., Puglisi, G., Lee, V.H., Fresta, M., 2003. Influence of preparation conditions on acyclovir-loaded poly-D,L-lactic acid nanospheres and effect of PEG coating on ocular drug bioavailability. Pharm. Res. 20, 584-590.

Gonzalez-Mira, E., Egea, M.A., Garcia, M.L., Souto, E.B., 2010. Design and ocular tolerance of flurbiprofen loaded ultrasound-engineered NLC. Colloids Surf. B 81 $412-421$.

Gonzalez-Mira, E., Egea, M.A., Souto, E.B., Calpena, A.C., Garcia, M.L., 2011. Optimizing flurbiprofen-loaded NLC by central composite factorial design for ocular delivery. Nanotechnology 22, 045101

Gonzalez, R.J., Tarloff, J.B., 2001. Evaluation of hepatic subcellular fractions for Alamar blue and MTT reductase activity. Toxicol. In Vitro 15, 257-259.

Hamid, R., Rotshteyn, Y., Rabadi, L., Parikh, R., Bullock, P., 2004. Comparison of alamar blue and MTT assays for high through-put screening. Toxicol. In Vitro 18 703-710.

Han, F., Li, S., Yin, R., Liu, H., Xu, L., 2008. Effect of surfactants on the formation and characterization of a new type of colloidal drug delivery system: nanostructured lipid carriers. Colloids Surf. A 315, 210-216.

Kawaguchi, E., Shimokawa, K.-i., Ishii, F., 2008. Physicochemical properties of structured phosphatidylcholine in drug carrier lipid emulsions for drug delivery systems. Colloids Surf. B 62, 130-135.

Lallemand, F., Daull, P., Benita, S., Buggage, R., Garrigue, J.S., 2012. Successfully improving ocular drug delivery using the cationic nanoemulsion, novasorb. J. Drug Deliv., 604204.

Liu, J., Huang, X.-f., Lu, L.-j., Li, M.-X., Xu, J.-c., Deng, H.-p., 2011. Turbiscan Lab ${ }^{\circledR}$ Expert analysis of the biological demulsification of a water-in-oil emulsion by two biodemulsifiers. J. Hazard. Mater. 190, 214-221.

Marianecci, C., Paolino, D., Celia, C., Fresta, M., Carafa, M., Alhaique, F., 2010. Nonionic surfactant vesicles in pulmonary glucocorticoid delivery: characterization and interaction with human lung fibroblasts. J. Control. Release 147, 127-135.

Metin, S., Hartel, R.W., 2005. Crystallization of fats and oils. In: Bailey's Industrial Oil and Fat Products. John Wiley \& Sons, Inc., New Jersey, USA.

Pignatello, R., Puglisi, G., 2011. Nanotechnology in ophthalmic drug delivery: a survey of recent developments and patenting activity. Recent Patents Nanomed. 1, 42-54.

Schubert, M.A., Harms, M., Müller-Goymann, C.C., 2006. Structural investigations on lipid nanoparticles containing high amounts of lecithin. Eur. J. Pharm. Sci. 27 226-236.

Schubert, M.A., Müller-Goymann, C.C., 2003. Solvent injection as a new approach for manufacturing lipid nanoparticles - evaluation of the method and process parameters. Eur. J. Pharm. Sci. 55, 125-131.

Severino, P., Andreani, T., Macedo, A., Fangueiro, J.F., Silva, A.M., Santana, M.H., Souto, E.B., 2012. Current state-of-art and new trends on 
lipid nanoparticles (SLN and NLC) for oral drug delivery. J. Drug Deliv., http://dx.doi.org/10.1155/2012/750891.

Shekunov, B.Y., Chattopadhyay, P., Tong, H.Y., Chow, A.H.L., 2007. Particle size analysis in pharmaceutics: principles, methods and applications. Pharm Res. 24, 203-227.

Souto, E.B., Doktorovova, S., Gonzalez-Mira, E., Egea, M.A., Garcia, M.L., 2010. Feasibility of lipid nanoparticles for ocular delivery of anti-inflammatory drugs. Curr. Eye Res. 35, 537-552.

Sultana, Y., Maurya, D.P., Iqbal, Z., Aqil, M., 2011. Nanotechnology in ocular delivery: current and future directions. Drugs Today (Barc) 47, 441-455.

Thoma, K., Serno, P., 1983. Thermoanalytischer Nachweis der Polymorphie der Suppositoriengrundlage Hartfett. Pharm. Ind. 45, 990-994.
Trotta, M., Pattarino, F., Ignoni, T., 2002. Stability of drug-carrier emulsions containing phosphatidylcholine mixtures. Eur. J. Pharm. Biopharm. 53, 203-208.

Vega, E., Gamisans, F., García, M.L., Chauvet, A., Lacoulonche, F., Egea, M.A., 2008 PLGA nanospheres for the ocular delivery of flurbiprofen: drug release and interactions. J. Pharm. Sci. 97, 5306-5317.

Ying, L., Tahara, K., Takeuchi, H., 2013. Drug delivery to the ocular posterior segment using lipid emulsion via eye drop administration: effect of emulsion formulations and surface modification. Int. J. Pharm. 453, 329-335.

Zimmer, A., Kreuter, J., 1995. Microspheres and nanoparticles used in ocular delivery systems. Adv. Drug Delivery Rev. 16, 61-73. 